\title{
Investigation of the engine-gear vortex with LIDAR and CFD
}

\section{Anton Stephan, Norman WIIdmann, I. N. Smalikho, F. Holzäpfel}

Anton Stephan, Norman WIIdmann, I. N. Smalikho, F. Holzäpfel, "Investigation of the engine-gear vortex with LIDAR and CFD

," Proc. SPIE 10833, 24th International Symposium on Atmospheric and Ocean Optics: Atmospheric Physics, 108336l (13 December 2018); doi: $10.1117 / 12.2504790$

SPIE Event: XXIV International Symposium, Atmospheric and Ocean Optics, Atmospheric Physics, 2018, Tomsk, Russian Federation 


\title{
Investigation of the engine-gear vortex with LIDAR and CFD
}

\author{
Anton Stephan ${ }^{\mathrm{a}}$, Norman Wildmann ${ }^{\mathrm{a}}$, I.N. Smalikho ${ }^{\mathrm{b}}$, and F. Holzäpfel ${ }^{\mathrm{a}}$ \\ aDLR Institute of Atmospheric Physics, 82234 Oberpfaffenhofen, Mnchner Str., 20, Germany \\ ${ }^{b}$ V.E. Zuev Institute of Atmospheric Optics, Siberian Branch of RAS, Academician Zuev \\ square, 634055 Tomsk, Russia
}

\begin{abstract}
The final approach and touchdown of a landing aircraft is studied numerically with a hybrid RANS/LES approach, as well as a pulsed coherent LIDAR system. Particular emphasis is put on the ground effect, the engine-gear vortex and the wake vortex evolution before and after touchdown. We compare CFD results with LIDAR measurement data collected in a recent campaign at Vienna airport. The radial velocity method was applied to retrieve vortex position and circulation. The vortex structures predicted by the simulations are clearly visible in those measurements. Simulated tangential velocity profiles are compared with vortex model profiles. It is observed that the standard vortex model can be improved including mirror vortices.
\end{abstract}

Keywords: LIDAR, wake vortex, computational fluid dynamics, engine-gear vortex

\section{INTRODUCTION}

Wake vortices are generated by flying aircraft in a natural way through the creation of lift. The complex wake structures in the aircraft's near-field roll-up to a system of two counter-rotating vortices further downstream. The generated two-vortex system can persist for several minutes, possessing a high amount of kinetic energy and thereby posing a potential hazard to following aircraft. In ground proximity the transport and decay of the wake vortices are mainly determined by the wind conditions and the interaction with the ground. To avoid wake-vortex encounters, regulatory separation distances between aircraft pairs have to be maintained based on the involved aircraft's weights, which limit the capacity of airports. Therefore, the investigation of wake-vortex decay is an important subject in aviation since the 1980s. ${ }^{1}$ A landing aircraft generates a highly complex flow field in terms of structure and relevant scales. The flow around the main wing, fuselage, slat, flap, jet engine and tailplane, as well as the interaction with the ground and the sudden lift reduction during touchdown substantially affect the generated wake vortices. ${ }^{2}$

Complementary to simulations, real wake vortex flows have mainly been investigated by measurements using Doppler wind LIDAR. ${ }^{3}$ An emitted laser beam is reflected and scattered by aerosols moving with the vortex flow. The detected signal provides the particle position and the phase shift of the photons, which reveals the velocity towards the detector by the Doppler-shift. A major challenge in experimental wake vortex physics is the development of accurate algorithms deducing the main vortex characteristics like position and circulation from the raw signal. It turns out that those algorithms are highly dependent on the LIDAR system and its technical specifications. For the well-established $1.54 \mathrm{~m}$ LIDAR systems the so-called radial velocity method (RVM) is yielding the best results. ${ }^{4}$ These algorithms are using a Burnham-Hallock vortex profile as a model vortex to estimate the vortex circulation from the radial velocity scans. In this work LIDAR measurements from a recent measurement campaign at Vienna international airport in November 2017 are analysed.

Further author information: (Send correspondence to Anton Stephan)

Anton Stephan: E-mail: anton.stephan@dlr.de, Telephone: +49 8153282566

24th International Symposium on Atmospheric and Ocean Optics: Atmospheric Physics, edited by Gennadii G. Matvienko, Oleg A. Romanovskii, Proc. of SPIE Vol. 10833, 108336I

(C) 2018 SPIE · CCC code: $0277-786 X / 18 / \$ 18 \cdot$ doi: $10.1117 / 12.2504790$

Proc. of SPIE Vol. 10833 108336I-1 


\section{LIDAR MEASUREMENT SETUP}

With the help of computational fluid dynamics we aim to investigate and interpret LIDAR measurements from a recent measurement campaign at Vienna international airport in November 2017. Pulsed coherent Doppler LIDARs (PCDLs) currently present a powerful technical instrument to study aircraft wake vortices. A $1.54 \mu \mathrm{m}$ Leosphere Windcube 200 was thus used during the campaign. The LIDAR was positioned about $280 \mathrm{~m}$ away from the flight corridor. A range-height indicator (RHI) scan pattern from $0^{\circ}$ to $20^{\circ}$ perpendicular to the flight path was implemented, see Fig. 1.

Table 1. Windcube 200s parameters.

\begin{tabular}{|l|l|}
\hline Wavelength $\lambda$ & $1.543 \mu \mathrm{m}$ \\
\hline Pulse energy $E_{p}$ & $50 \mu \mathrm{J}$ \\
\hline Pulse duration $\tau_{p}$ & $200 \mathrm{~ns}$ \\
\hline Pulse repetition frequency $f_{p}$ & $20 \mathrm{kHz}$ \\
\hline Intermidiate frequency & $69.3 \mathrm{MHz}$ \\
\hline Sampling rate & $250 \mathrm{MHz}$ \\
\hline Time window $T_{W}$ & $140 \mathrm{~ns}$ \\
\hline Physical resolution $\Delta z$ & $39.4 \mathrm{~m}$ \\
\hline Band width & $0-125 \mathrm{MHz}$ \\
\hline Channel number & 32 \\
\hline Resolution & $3 \mathrm{~m} / \mathrm{s}$ \\
\hline Pulse number $N_{a}$ & 2000 \\
\hline Minimum range & $100 \mathrm{~m}$ \\
\hline Range gate length $R$ & $3 \mathrm{~m}$ \\
\hline Maximum elevation angle $\phi$ & $20 \mathrm{deg}$ \\
\hline Scanning rate & $4 \mathrm{deg} / \mathrm{s}$ \\
\hline Distance to runway & $300 \mathrm{~m}$ \\
\hline
\end{tabular}

Figure 1 depicts the scanning geometry that was used during the measurement campaign. In total more than 200 aircraft overflights have been measured. The general theory of that kind of LIDARs and post-processing algorithms can be found in ${ }^{4}$ and. ${ }^{5}$

\section{CFD METHODS}

\subsection{RANS methods - model, mesh, solver}

Within the DLR project L-bows an Airbus A320 airplane was used as a wake vortex generator. For the RANS simulations a very detailed CAD model of the Airbus A320 "ATRA" operated by the German Aerospace Center (DLR) was available. All investigations shown in this paper were carried out using the high-lift configuration "Full LDG". This implies a deflection angle of $\delta_{\text {flaps }}=40^{\circ}$ for the flaps and a deflection angle of $\delta_{\text {slats }}=27^{\circ}$ for the slats. Besides the high-lift elements, the CAD model also includes the slat tracks, the IAE V2500 turbofan engine cowling, the flap track fairings and the vertical and horizontal tail planes. Furthermore, the CAD model also includes a slightly simplified main and nose landing gear. Fig. 2 (a) gives an overview of the CAD model used. In Fig. 2 (b) the CAD model of the simplified main landing gear is shown. The RANS simulations were performed with and without landing gear.

The RANS simulations within the project L-bows were performed using hybrid unstructured meshes. The meshes were build using the mesh generator Centaur, ${ }^{6}$ Additionally, the volume meshes were strongly refined 


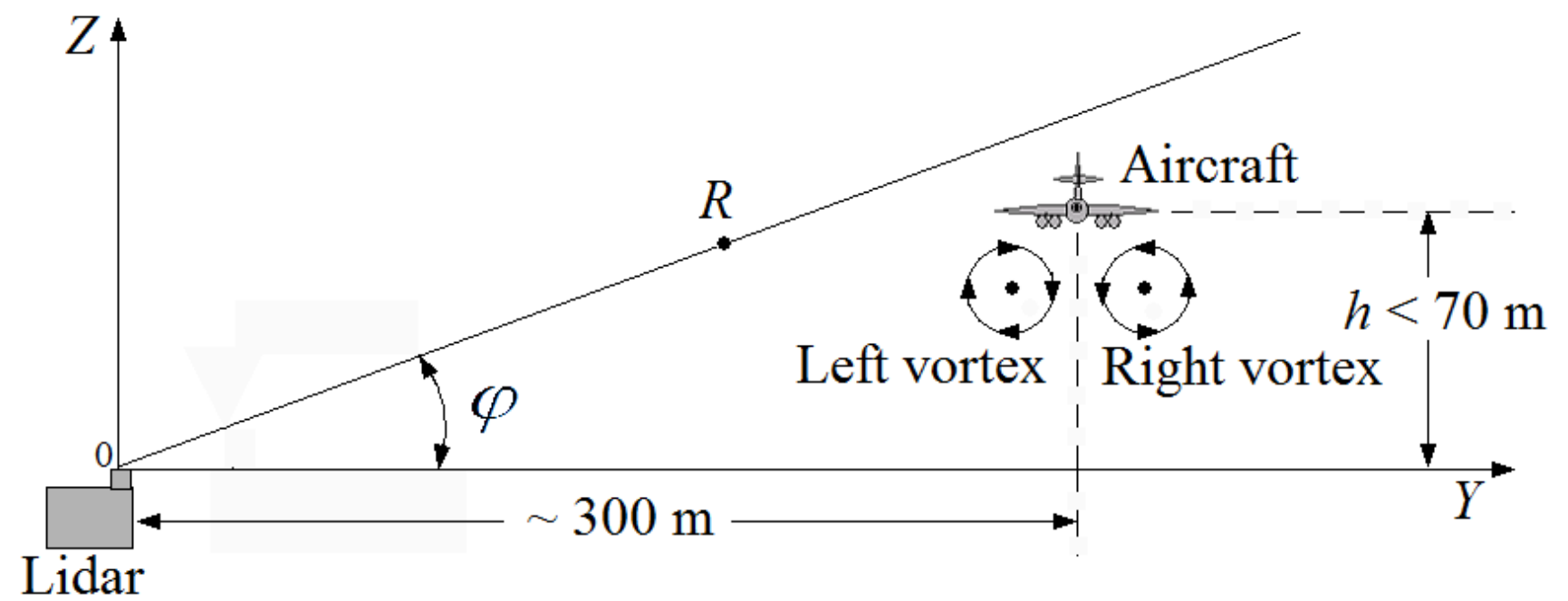

Figure 1. Geometry of RHI scan in the vertical plane.

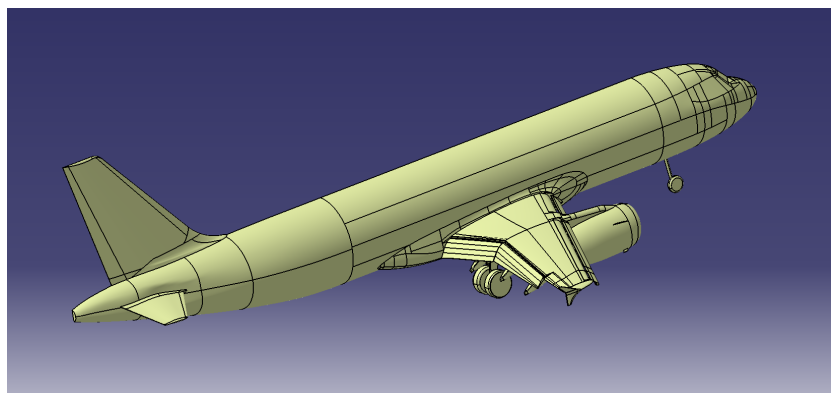

(a) Overview

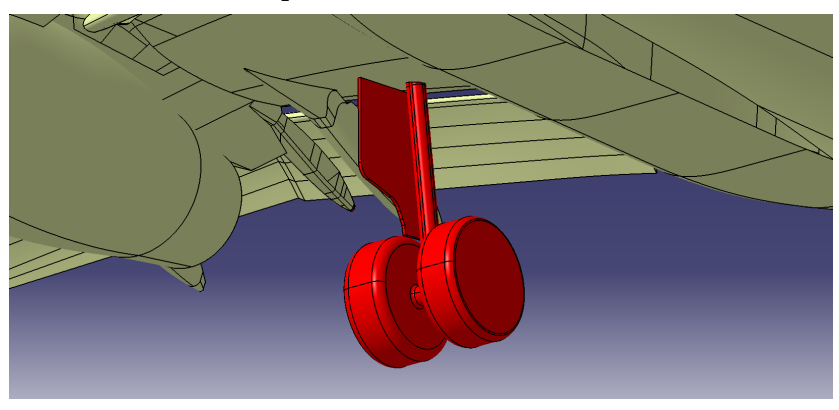

(b) Main landing gear

Figure 2. CAD model

in the regions of wakes and vortex systems produced by the wing, the high-lift devices, the landing gears and the tail planes. To achieve a proper resolution of the boundary layers on the non-slip walls, 40 prisms layers were used on all non-slip walls in the complete mesh. The meshes are, thereby, of a very high resolution in order to resolve all relevant flow phenomena around the Airbus A320 high-lift configuration. Figure 3 (a) provides an overview of the Centaur volume source setup.

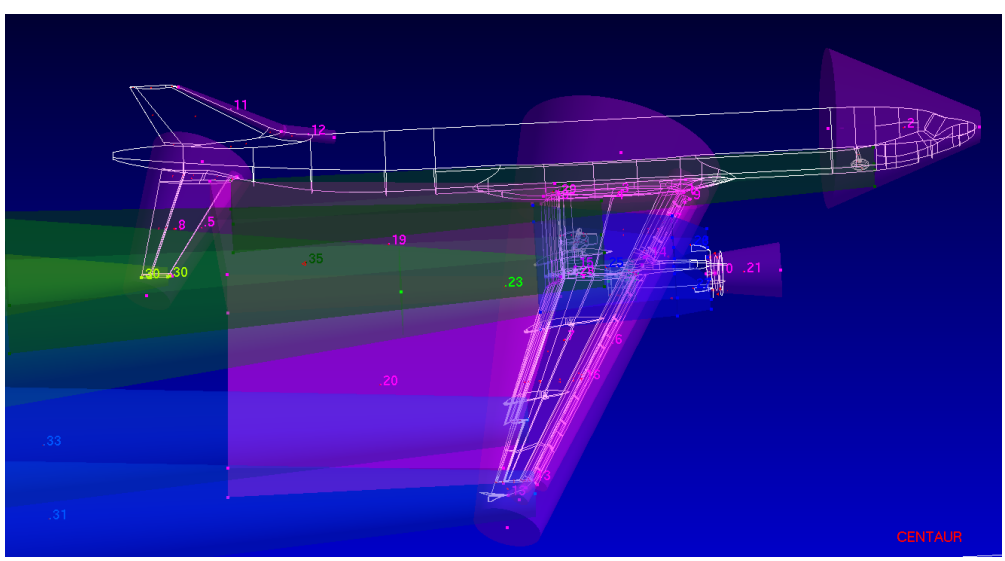

(a) Centaur setup

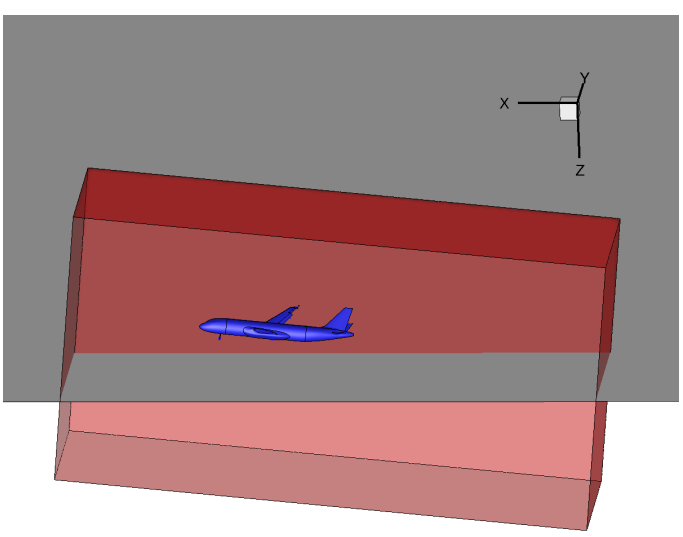

(b) Block setup

Figure 3. Overview of volume source and mesh block setup 


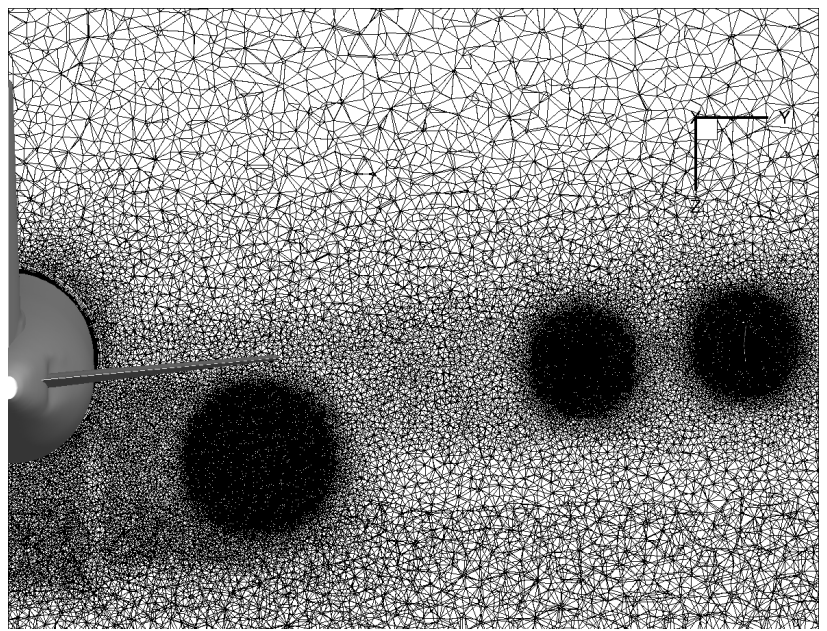

(a) Before automatic refinement

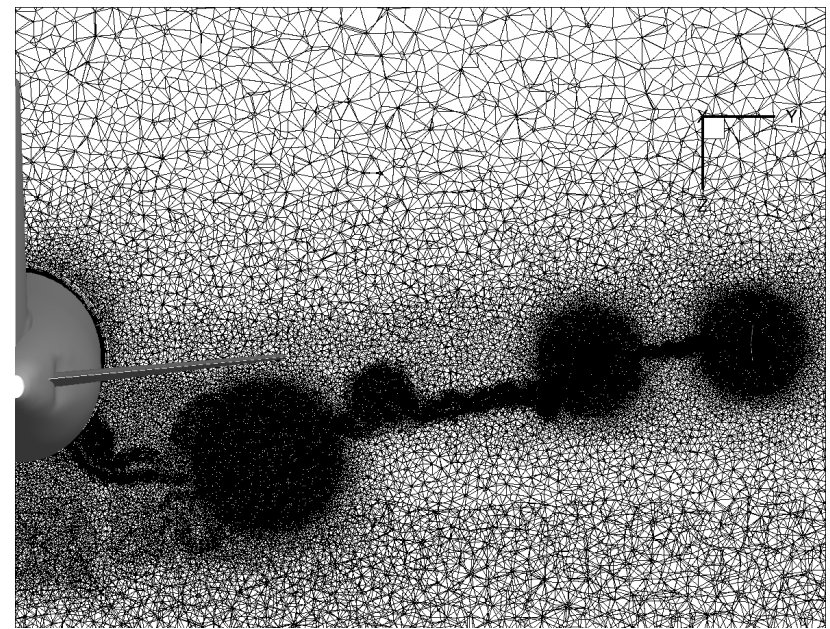

(b) After automatic refinement

Figure 4. Slice through mesh

To realize the simulation at different altitude steps, an approach using multiple mesh blocks was chosen for the meshing. The simulated flow volume is divided in several overlapping mesh blocks, which are meshed separately. After the meshing, the relative position of the blocks is set up in the flow solver and the flow field is calculated for the whole domain using the Chimera technique for the exchange of flow data between the mesh blocks. By using this meshing approach, it is possible to build one separate mesh block for the aircraft and one separate mesh block for the far field including the ground. The relative positioning of these blocks enables realizing different altitude steps by the use of identical meshes. In Fig. 3 (b) an overview of the mesh block setup for one altitude step is given. All mesh parts which are not needed for the simulation, like the mesh below the ground or behind the aircraft, are blanked by the flow solver.

To further improve the quality of the simulated flow field around the aircraft configuration, at each altitude step the meshes were locally refined during the simulation process. This was done by an automatic mesh adaptation process provided by the flow solver. The mesh adaptation is based on a user defined sensor function and is restricted to user defined regions in the flow field. In the simulations presented in this paper, the mesh adaptation was locally restricted to the regions around the vortices and the wakes of the configuration. As a sensor function, the difference in total pressure between two neighboring mesh points was set. The meshes were adapted in up to nine stages for both configurations, with and without gears, for all altitude steps. In Fig. 4 (a) a slice through the volume mesh before the first and in Fig. 4 (b) after nine adaptation steps is shown. An improvement of the mesh resolution in the wake of the wing and around the tip vortices of the wing and the high-lift devices can clearly be seen.

Within the current studies the DLR TAU Code ${ }^{7}$ was used for the RANS and URANS simulations. TAU is a compressible, unstructured finite volume solver for the Reynolds-averaged Navier-Stokes equations. For all simulations shown in this paper, the Spalart-Allmaras (SA) turbulence model ${ }^{8}$ was used. The SA model is robust and sufficiently validated for the simulation of the flow around complex high-lift configurations. ${ }^{9-11}$ The time-stepping was done by an implicit lower-upper symmetric Gauß-Seidel (LU-SGS) scheme. A central second-order scheme with Matrix dissipation was employed. A standard "3v" multi-grid cycle was applied to accelerate convergence.

\subsection{Flow conditions}

The used flow conditions are derived from a real touchdown maneuver of the Airbus A320 "ATRA". They were converted to the International Standard Atmosphere (ISA) at an altitude of $h=0 \mathrm{~m}$. The resulting flow conditions for the simulations are shown in Tab. 2. 
Table 2. Flow conditions

\begin{tabular}{|l|r|}
\hline quantity & $\rho=1.225 \mathrm{~kg} / \mathrm{m}^{3}$ \\
\hline Density & $p=101325 \mathrm{~Pa}$ \\
\hline Pressure & $T=288.15 \mathrm{~K}$ \\
\hline Temperature & $U_{\infty}=64 \mathrm{~m} / \mathrm{s}$ \\
\hline Velocity & $R e \approx 18.4 * 10^{6}$ \\
\hline Reynolds number & $M a \approx 0.188$ \\
\hline Mach number & $b=34.1 \mathrm{~m}$ \\
\hline wing span & $A=122.6 \mathrm{~m}{ }^{2}$ \\
\hline wing plan-form area & $C_{L}=1.67$ \\
\hline total lift coefficient & \\
\hline
\end{tabular}

The reference values for an elliptic load distribution, ${ }^{1}$ initial circulation, vortex spacing, vortex descent velocity, characteristic time, vorticity unit are given as

$$
\Gamma_{0}=\frac{2 C_{L} U_{\infty} A}{\pi b}, \quad b_{0}=\frac{\pi}{4} b, \quad w_{0}=\frac{\Gamma_{0}}{2 \pi b_{0}}, \quad t_{0}=\frac{b_{0}}{w_{0}}, \quad \omega_{0}=\frac{1}{t_{0}},
$$

resulting in $\Gamma_{0}=244.2 \mathrm{~m}^{2} / \mathrm{s}, b_{0}=26.8 \mathrm{~m}, w_{0}=1.45 \mathrm{~m} / \mathrm{s}$, and $t_{0}=18.45 \mathrm{~s}$, see Tab. 3 . For the sake of clarity physical quantities are not normalized in this work. We set $t=0$ at the instant of the touchdown or the crossing of a surface, depending on the context. The maximum tangential velocity of the wake vortices is around $20 \mathrm{~m} / \mathrm{s}$.

Table 3. Reference values

\begin{tabular}{|l|r|}
\hline quantity & $b_{0}=26.8 \mathrm{~m}$ \\
\hline initial vortex separation & $\Gamma_{0}=244.2 \mathrm{~m}^{2} / \mathrm{s}$ \\
\hline initial circulation & $w_{0}=1.45 \mathrm{~m} / \mathrm{s}$ \\
\hline vortex descent speed & $t_{0}=18.45 \mathrm{~s}$ \\
\hline characteristic time & \\
\hline
\end{tabular}

The steady RANS simulations were performed for the configurations with and without landing gears at a constant angle of attack for eight altitude steps between $h=0 \mathrm{~m}$ and $h=175 \mathrm{~m}$. In this study the altitude is defined as the distance between the main landing gear and the ground. The angle of attack was set to $\alpha=6^{\circ}$, which was the average angle of attack during the approach in the flight test data.

\subsection{LES methods}

The Large Eddy Simulation is performed using the incompressible LES code MGLET, developed at the Technical University of Munich, ${ }^{12}$ It solves the incompressible Navier-Stokes equations:

$$
\begin{aligned}
\frac{\partial u_{i}}{\partial t}+\frac{\partial\left(u_{i} u_{j}\right)}{\partial x_{j}} & =-\frac{1}{\rho} \frac{\partial p^{\prime}}{\partial x_{i}}+\frac{\partial}{\partial x_{j}}\left(\left(\nu+\nu_{t}\right) 2 S_{i j}\right), \\
\frac{\partial u_{j}}{\partial x_{j}} & =0 .
\end{aligned}
$$

Here $u_{i}$ represents the velocity components in the three spatial directions $(i=1,2$, or 3$), S_{i j}=\left(\partial u_{i} / \partial x_{j}+\right.$ $\left.\partial u_{j} / \partial x_{i}\right) / 2$ denotes the strain rate tensor and $p^{\prime}=p-p_{0}$ equals the pressure deviation from a reference state $p_{0}$. The kinematic viscosity is given as the sum of molecular viscosity $\nu$ and eddy viscosity $\nu_{t}$, determined by means of a Lagrangian dynamic sub-grid scale model,. ${ }^{13}$ Eqns. (2) and (3) are solved by a finite-volume approach, using a fourth-order compact scheme,. ${ }^{14} \mathrm{~A}$ split-interface algorithm is used for the parallel solution of the tri-diagonal system, resulting from the compact scheme. A third-order explicit Runge-Kutta method is used for the time integration. The simulations are performed in parallel, using a domain decomposition approach.

A realistic aircraft wake is generated in an LES domain by sweeping a high-fidelity steady RANS flow field through the domain, which enables the simulation of the wake-vortex evolution from its generation until its final 
decay. ${ }^{15}$ The RANS flow field delivers a forcing term for the Navier-Stokes equations solved in the LES. This approach is referred to as a fortified solution algorithm, ${ }^{16}$ or a nudging technique used in data assimilation. ${ }^{17}$ The resulting velocity field in the aircraft vicinity consists of the weighted sum

$$
\mathbf{V}=f(y) \mathbf{V}_{\mathrm{LES}}+(1-f(y)) \mathbf{V}_{\mathrm{RANS}}
$$

of the LES and the RANS velocity fields, see Figure 5, with a transition function

$$
f(y, \alpha, \beta)=\frac{1}{2}\left[\tanh \left[\alpha\left(\frac{y}{\beta}-\frac{\beta}{y}\right)\right]+1.0\right] .
$$

Here $\alpha$ and $\beta$ represent the slope and the maximum wall-distance of the transition region, for which we employ

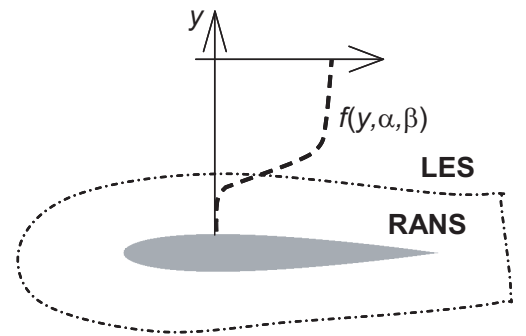

Figure 5. Schematic of a weighting function for a combination of RANS and LES flow fields. ${ }^{15}$

similar values as in. ${ }^{15}$ This hybrid method also enables us to study the effect of an ambient turbulent crosswind on the aircraft wake by initializing the wake within a pre-simulated turbulent wind field, ${ }^{18}$ In the present work stratification effects are not taken into account.

\section{SIMULATION RESULTS}

\subsection{Flow field}

The evolution of the coherent structures in the wake shall be worked out in Fig. 6. The axial vorticity is depicted in a slice perpendicular to the runway at $x=50 \mathrm{~m}$. The aircraft is passing the slice $6.4 \mathrm{~s}$ before touchdown. Right after the wings crossed the slice $(t=-6.4 \mathrm{~s})$ we observe vorticity sheets behind them, with the wing and flap tip vortices becoming apparent, as well as vorticity shedding off the engine, slats and gears in Fig. 6 (a). The fuselage creates counter-rotating vorticity. After $0.2 \mathrm{~s}$ the tail wings cross the slice. The counter-rotating tailplane and fuselage-wing junction vortices are clearly visible. The main vortices have already established themselves. Note that one single vortex patch is forming behind the engine. In the sequel the complex multi-vortex system is further developing. The coherency of the engine vortex is strongly pronounced, Fig. 6 (d),(e), evolving from the fuselage wake.

The wing and flap tip vortex merge quickly and descend, Fig. 6 (e)-(i), the inner vortices are transported by the vorticity of the main vortices. The fuselage junction vortices dissipate quickly. Remarkably, the engine vortices as well as the tailplane vortices stay coherent and are transported in the vortex field. Due to the ground and the main vortices, the coherent engine vortices are transported quickly outboard in spanwise direction and even start to rebound from the ground, Fig. 6 (g) -(i). * We also observe a secondary vorticity layer emerging. Finally, we have three coherent vortices at $t=3.7 \mathrm{~s}$ in the case with gears, which slowly merge in the sequel and we observe only one in the simulations without landing gear. As we will see, this effect translates to a stronger overall circulation reduction. On the one hand it is known that the behavior of multiple interacting vortices may substantially differ for slightly different vortex strengths and positions. On the other hand similar vortex structures can also be observed in LIDAR measurements, see Sec. 5, which provides evidence that the observed vortex constellation may occur.

${ }^{*}$ This effect of two vortices rolling-up close to the ground is thoroughly investigated in ${ }^{2}$ for the wing and flap tip vortices of an A340 aircraft. 


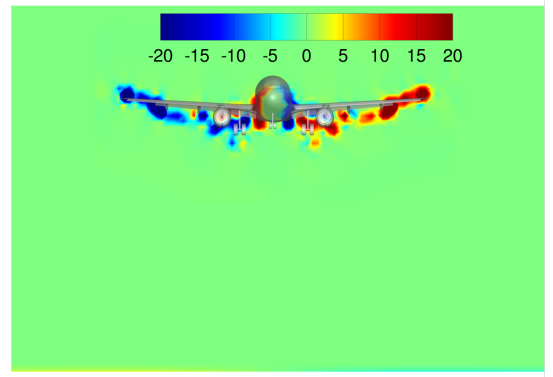

(a) $t=-6.4 \mathrm{~s}$

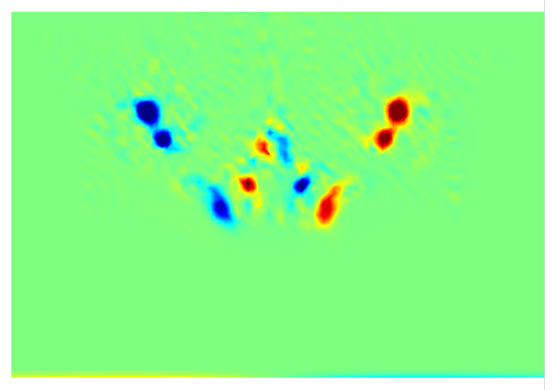

(d) $t=-5.0 \mathrm{~s}$

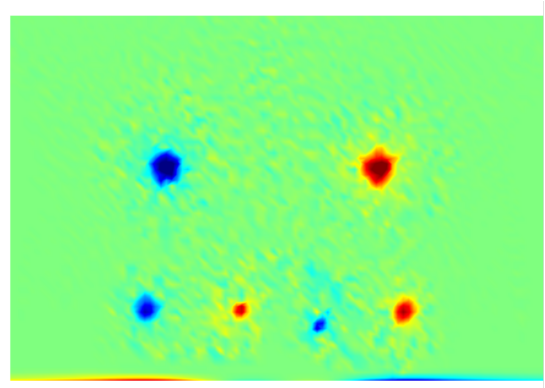

(g) $t=-0.4 \mathrm{~s}$

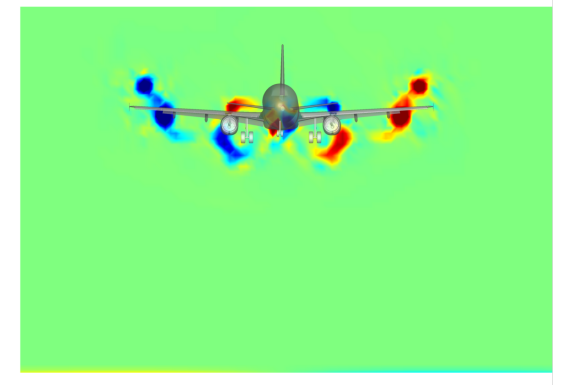

(b) $t=-6.2 \mathrm{~s}$

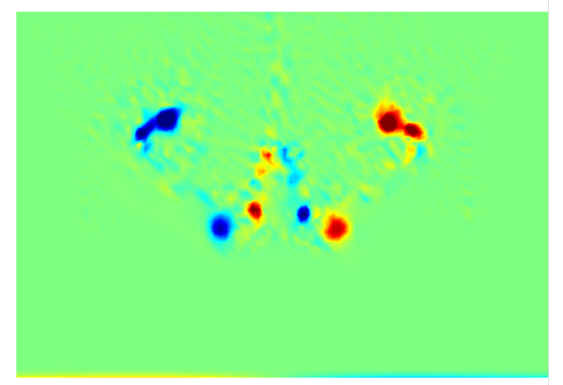

(e) $t=-4.5 \mathrm{~s}$

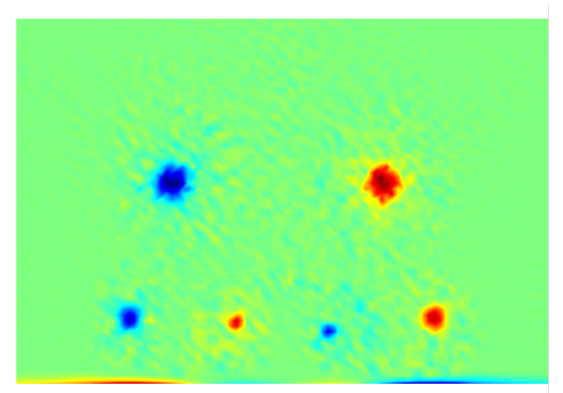

(h) $t=0.5 \mathrm{~s}$

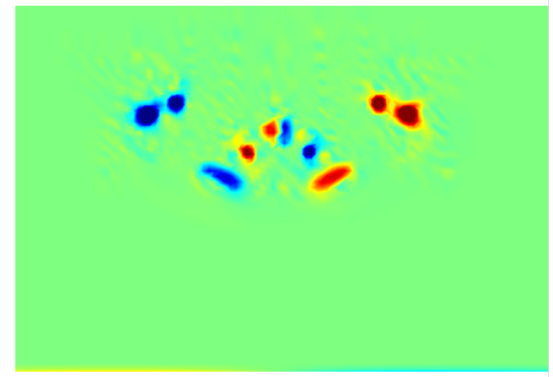

(c) $t=-5.4 \mathrm{~s}$

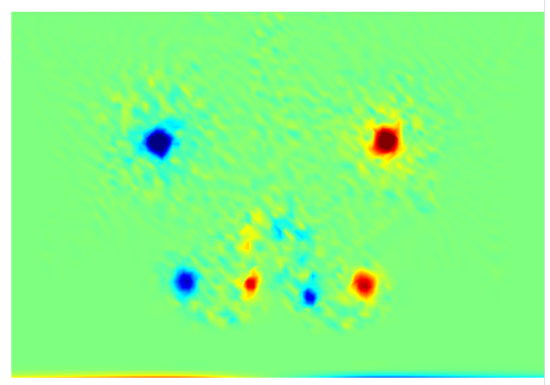

(f) $t=-2.4 \mathrm{~s}$

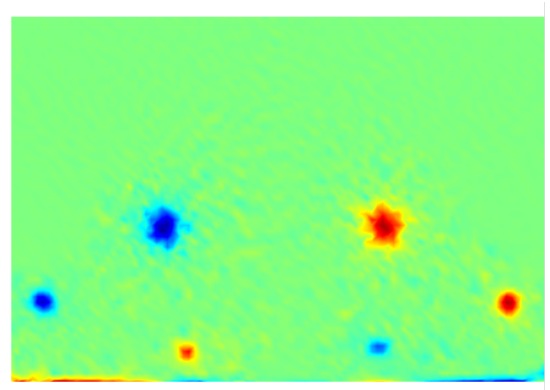

(i) $t=3.7 \mathrm{~s}$

Figure 6. Video $1 \omega_{x}$ distribution - a slice where the aircraft with landing gears is passing through $6.4 \mathrm{~s}$ before touch-down. http://dx.doi.org/10.1117/12.2504790 


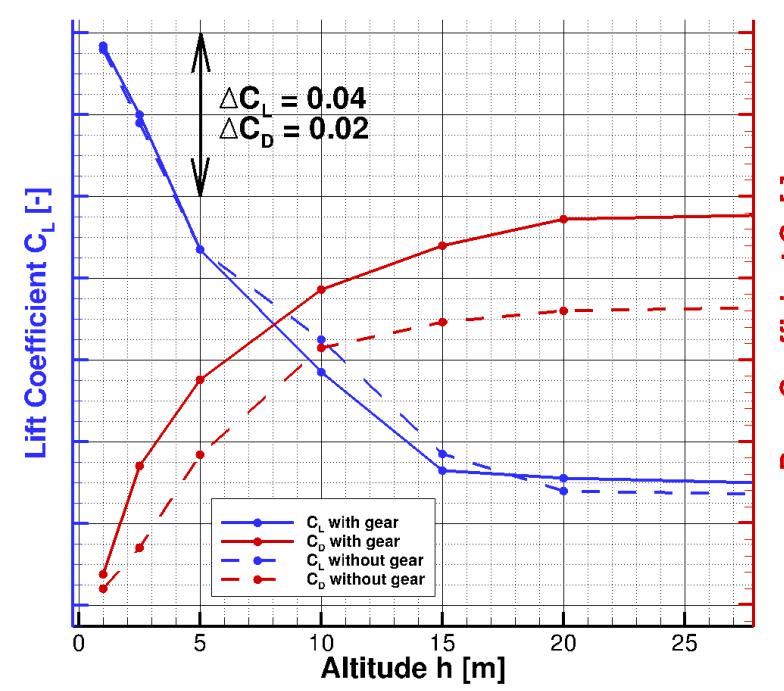

(a) Influence of altitude and landing gear on $C_{L}$ and $C_{D}$.

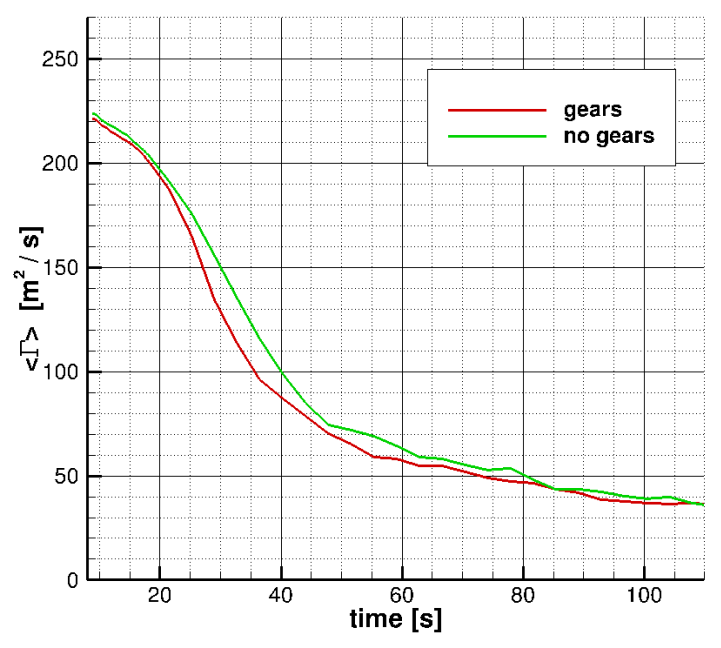

(b) Circulation decay averaged

Figure 7. Landing gear effect on $C_{L}, C_{D}$ and circulation.

\subsection{Gear effect on aerodynamics and vortex evolution}

In Fig. 7 (a) the influence of the ground effect and the landing gear on the lift and drag coefficients is shown for the simulated altitudes. According to Fig. 7 (a) the landing gear has no significant influence on the lift coefficient of the Airbus A320 in high-lift configuration for all considered altitude steps. In contrast, the influence of the landing gear on the drag coefficient is clearly visible for all considered altitude steps. The landing gear accounts for an isolated local disturbance of the flow field around the wing and thus has only a minor impact on the lift coefficient. The small disturbance is located only at the lower side of the inner main wing near the nose of the fuselage. Most of the wing, especially its upper side, the fuselage and the tail planes are not influenced by the landing gear. Furthermore, the surfaces of the landing gear itself are not producing a substantial amount of lift.

In contrast to the minor influence of the landing gear on the lift, the drag is significantly increased by the addition of the landing gear for all altitude steps. This increase is also localized at the landing gear itself since, as described above, the mean flow around the aircraft configuration is only weakly influenced by the landing gear. The landing gear can be considered as a large blunt body located in nearly undisturbed flow producing a strong wake, but almost no lift. The influence of the landing gear on the drag coefficient is decreasing with decreasing altitude. This can be explained by the growing influence of the ground effect on the flow in the surrounding area with decreasing altitude. The growing influence of the ground effect results in a reduced local flow velocity, especially at the position of the main landing gear below the wing, resulting in reduced drag for the whole aircraft configuration for decreasing altitudes. The long-time development of the circulation and the effect of the landing gear are depicted in Fig. 7 (b). Here we average the circulation over the first $400 \mathrm{~m}$, where the vortices do not link with the ground. A slightly faster decay is observed when using in the simulations with landing gears. This can be explained by the coherency of the engine-gear-vortex leading to a stronger vortex-ground interaction and a more turbulent decay phase. However, this effect is moderate.

\subsection{Tangential velocity profiles}

In this section we analyze tangential velocity profiles, $V_{\theta}$, in different slices along the glide path and compare them to the widely used Burnham-Hallock vortex model,

$$
V_{\theta}=\frac{\Gamma_{0}}{2 \pi r} \frac{r^{2}}{r^{2}+r_{0}^{2}}
$$

where $r_{0}$ denotes the core radius. Figure 8 depicts $V_{\theta}$ profiles for different times after crossing planes at different 


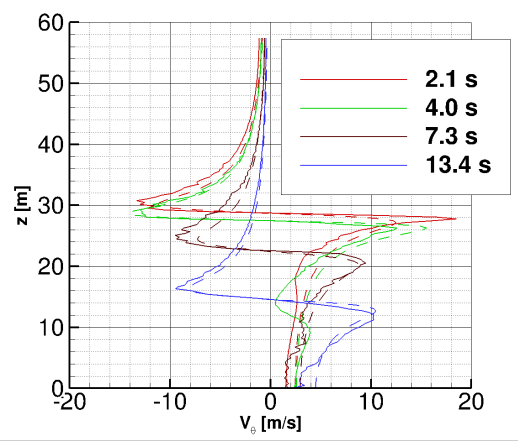

(a) gears, $x=2 \mathrm{~m}$

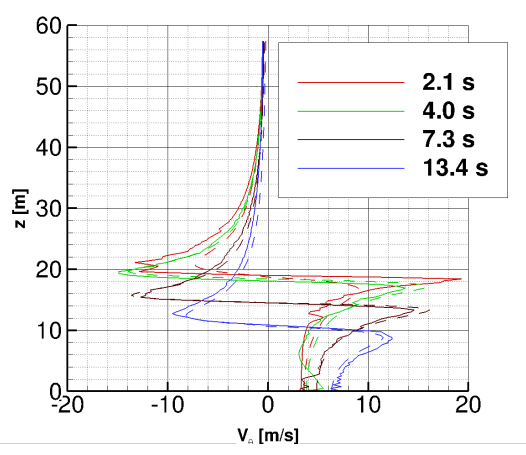

(b) gears, $x=154 \mathrm{~m}$

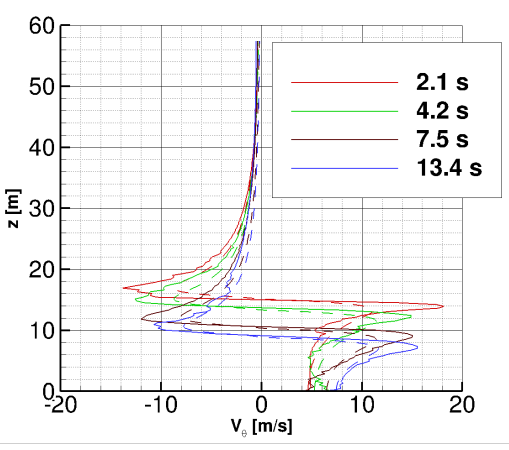

(c) gears, $x=231 \mathrm{~m}$

Figure 8. Tangential velocity profiles compared to Burnham-Hallock model including mirror vortices.

planes in space, i.e. distances to the touchdown point. Note that we do not observe an anti-symmetric velocity profile, as the aircraft approaches the ground. This effect is mostly captured by the assumption of mirror vortices that model the effect of the wall. Therefore we compare all profiles with the Burnham-Hallock vortex model including a mirror vortex. The effect of the neighboring vortex can be neglected, since we only consider the horizontal velocity components.

This model fits very well at sufficiently for most situations, Fig. 8 (a) to (c). Note the influence of the engine-gear vortex in the simulation with landing gears after crossing. Particularly very close to the ground, the Burnham-Hallock vortex approximation is getting better with time. The influence of the landing gear is not apparent after $10 \mathrm{~s}$. We observe maximum velocities at the ground surface of about $8 \mathrm{~m} / \mathrm{s}$. Indeed, vortex models are widely used for the post-processing of LIDAR wake vortex measurements, ${ }^{4,19}$ Especially the Burnham-Hallock model is used in an iterative process that minimizes the error to a certain model field. The presented results indicate that those models should be improved in ground proximity by using mirror vortices. Even the use of mirror vortices in the present algorithms does not entirely meet the character of the flow field very close to the ground when other vortices, like the engine-gear vortex are sufficiently coherent.

\subsection{Validation with flight test data}

To study the flare and touchdown phases we employ flight test data from tests that have been performed by the Institute of Aerodynamics and Flow Technology (DLR) as validation experiments for the simulation model A320-ATRA on behalf of Airbus. The present flight test data of a landing (test No.12) was kindly provided by Christian Raab. Here we compare the simulation results with flight measurement data in terms of circulation. We use different definitions for the circulation in the subsequent discussion. All of those are evaluated in the LES domain. First, $\Gamma_{0}$ as it is defined in Eq. (1) at different aircraft positions, depending on the lift coefficient $C_{L}$. Second, a scaled version of the widely used $\Gamma_{5-15}$ is employed, namely

$$
\Gamma_{3-9}=\frac{1}{6} \int_{3 \mathrm{~m}}^{9 \mathrm{~m}} \Gamma(r) d r
$$

where $\Gamma(r)=\int_{D_{r}} \omega_{x} \cdot d A$ denotes the circulation in a disc of radius $r$ centered in the vortex core, which is evaluated in the flow field of the simulation. Third, we use $\Gamma_{H S}=\int_{H S} \omega_{x} \cdot d A$, the integral of the vorticity in flight direction, taken over the entire half-space of the simulated domain.

The circulation derived from flight data is slightly increasing during flare and strongly increasing shortly before touchdown where we actually cannot expect an elliptical wing loading. So, the instant before touchdown has to be analyzed carefully. As we do not have flight data statistics, we cannot consider the flight data graph as the only acceptable truth, but qualitative and quantitative behavior agree well. All curves are slightly increasing until shortly before touchdown. After that the area used to calculate $\Gamma_{3-9}$ is overlapping with the ground. Here, the respective disc segment above ground is used for integration. However, the developing secondary vorticity boundary layer at the ground leads to a reduction of $\Gamma_{3-9}$. 


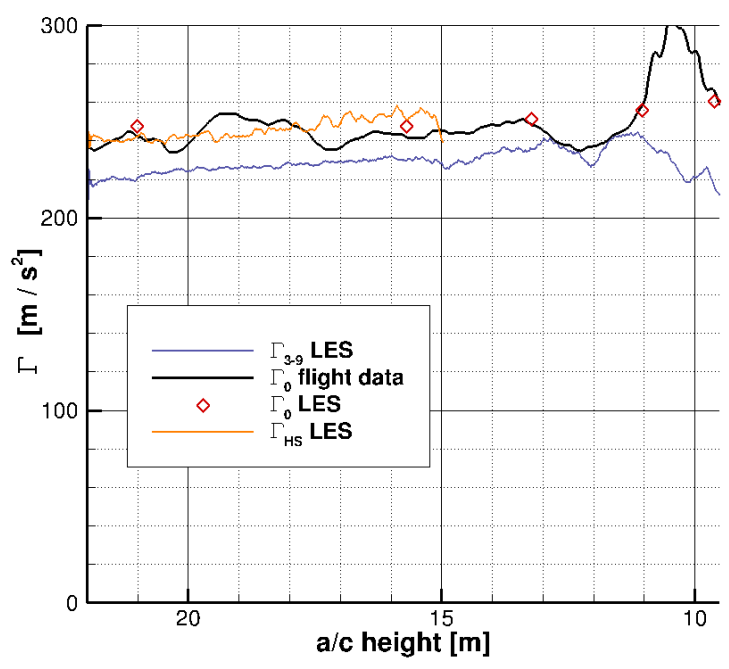

Figure 9. Circulation development for different hybrid techniques compared with flight data along the glide path.

It turns out that $\Gamma_{3-9}$ does only capture about $90 \%$ of the entire circulation, created by the aircraft, which is in agreement with former results, ${ }^{2,15}$ indicating that vorticity patches may be distributed over relatively large areas. Due to the developing circulation boundary layer, $\Gamma_{H S}$ does not show sensible results very close to the ground, therefore it is omitted in Fig. 9 for $x>225 \mathrm{~m}$. Finally, $\Gamma_{0}$ from the landing experiments is plotted in Fig. 9, showing a very consistent behavior with $\Gamma_{0}$ for simulations.

\section{LIDAR MEASUREMENTS}

The raw LIDAR data consists of Doppler velocity spectra for every point in the scanning plane. A straightforward retrieval of that kind of data employs the radial velocity component of the flow field towards the LIDAR, which is depicted in Fig. 10. In Fig. 10 we observe RHi scans for different time steps after a B747 has crossed the measurement plane, starting at a vortex age of $t=42 \mathrm{~s}$. The plots depict the radial velocity $V_{r}$ along the laser beam. Positive velocities point away from the LIDAR. The wake vortex system leads to a characteristic quadrupole pattern. We observe how the downwind vortex is advected towards the LIDAR, whereas the upwind vortex hovers above the initial position. In Fig. 10 (b) and (c) we clearly see blue streaks from the downwind vortex towards the LIDAR, that indicate the presence of another smaller vortex. Particularly significant is the outflow of the positive radial velocity of the upwind vortex in Fig. 10 (c) to (e). At a time of $t=72 \mathrm{~s}$ that outflow suddenly vanishes, see Fig. 10 (f). We would not expect that flow field pattern generated by just one coherent vortex.

The RVM is applied to the LIDAR measurements. Figure 11 (a) and (b) depict the circulation and vortex trajectories, respectively. As in Fig. 2 the upwind vortex hovers above the runway. In the circulation plot sudden falls and reorganizations can be observed, right in the beginning as well as after 60 and 120 seconds. From the previous investigations this might not be physical, but instead reflect the fact that there is a multi-vortex system at those times. In the beginning the roll-up of the wing tip and flap tip vortex, and after 60 seconds the presence of the engine-gear vortex. Generally speaking, the described effects cannot be observed in all cases, since they depend on the aircraft type and are more pronounced for larger aircraft. This is partly an effect of the LIDAR resolution, but has to be investigated further.

\section{COMPARISON OF CFD AND LIDAR RESULTS}

The LIDAR images can be simulated by calculating the radial velocity that a LIDAR would observe. Figure 12 (a) depicts five instantaneous scans at different distances to the touchdown point, for two time steps after touchdown. As we do not include wind in these simulations we have an almost symmetric field, though the 


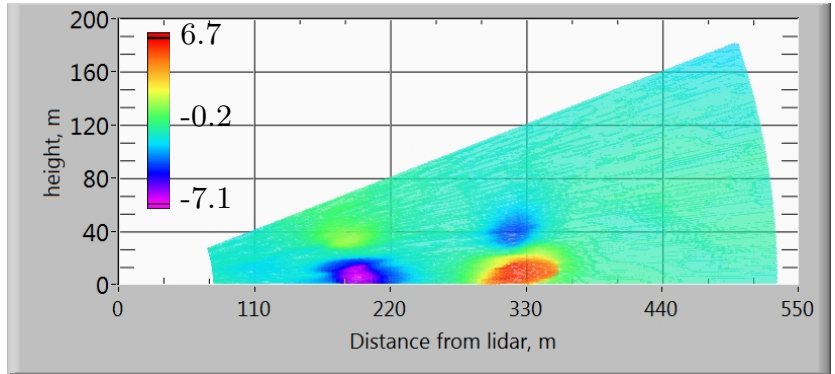

(a) $t=42 \mathrm{~s}$

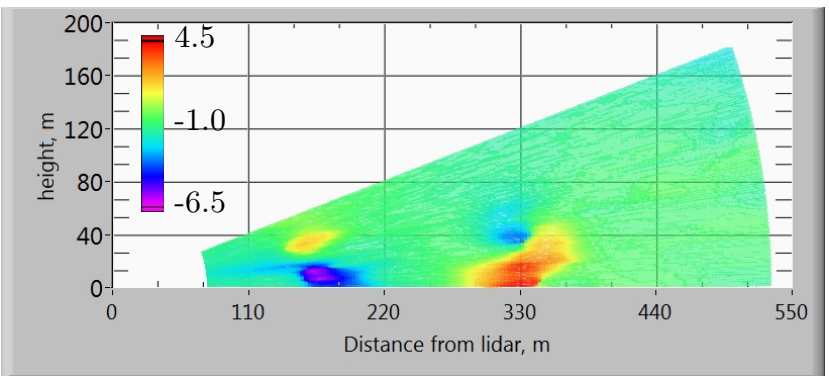

(c) $t=54 \mathrm{~s}$

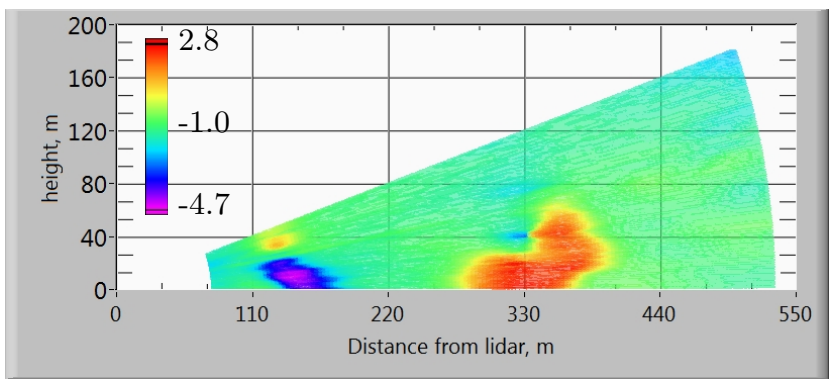

(e) $t=66 \mathrm{~s}$

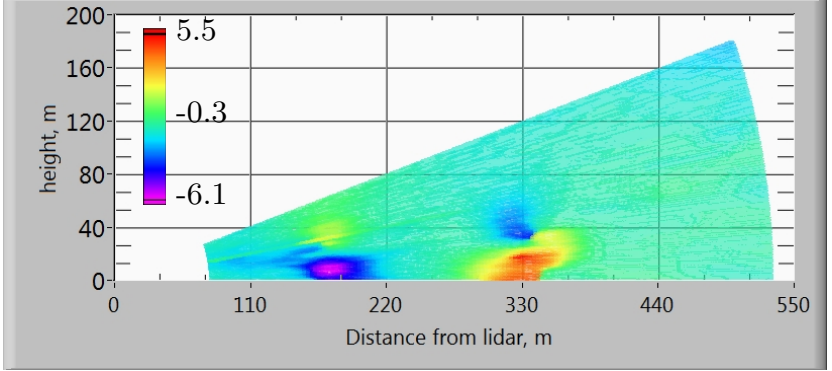

(b) $t=48 \mathrm{~s}$

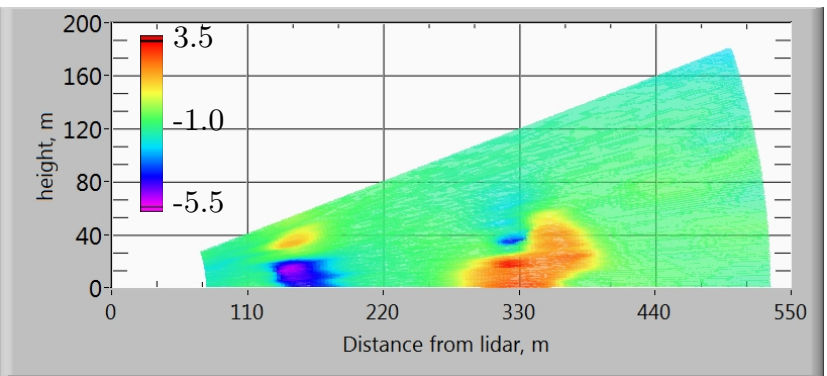

(d) $t=60 \mathrm{~s}$

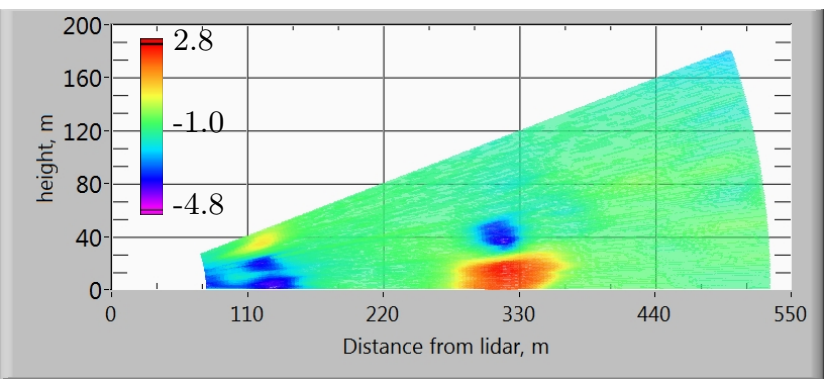

(f) $t=72 \mathrm{~s}$

Figure 10. LIDAR RHi scan, radial velocity component in $\mathrm{m} / \mathrm{s}$, different times after the passage of a B747 aircraft

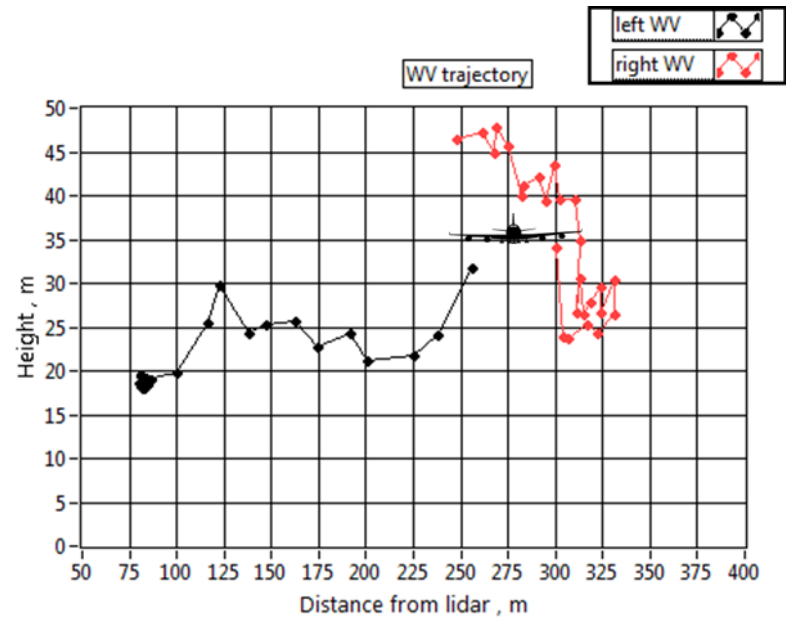

(a) $t=6.5 \mathrm{~s}$

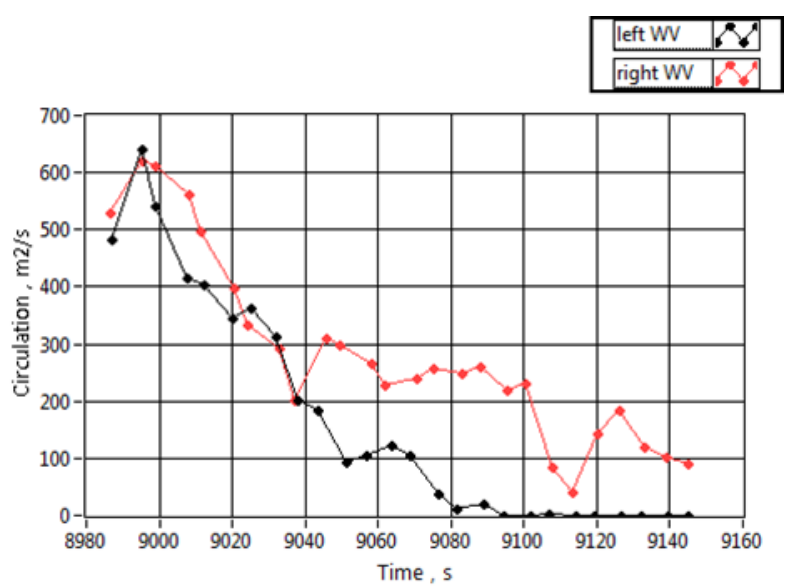

(b) $t=13 \mathrm{~s}$

Figure 11. Vortex circulation development and trajectories of a B747, obtained from LIDAR measurements with the use of the RVM. 


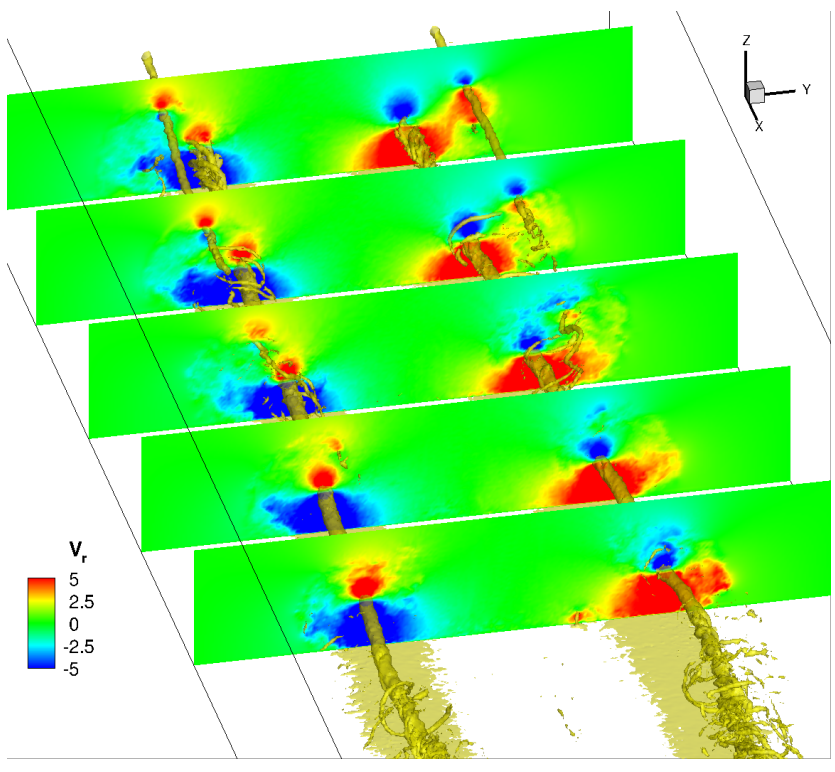

(a) slices behind runway

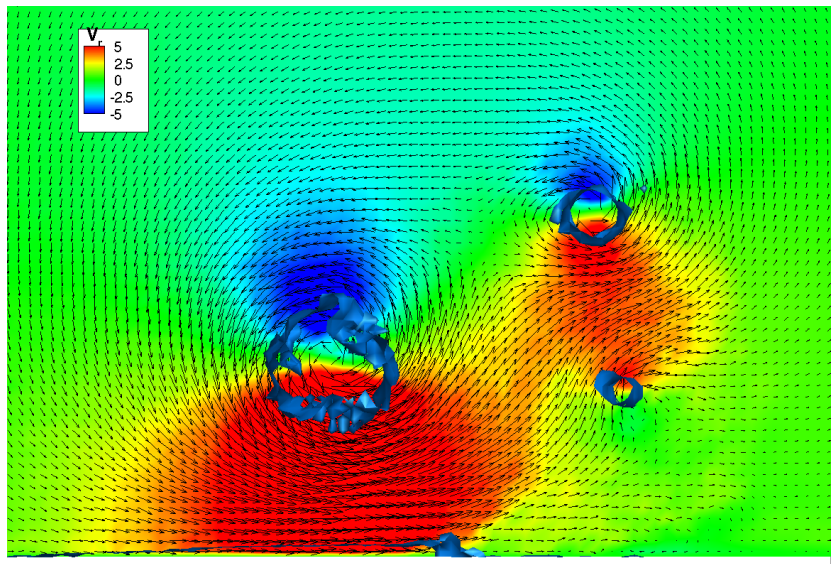

(b) close-up with involved vortices

Figure 12. Simulated LIDAR RHi scan, radial velocity, vorticity magnitude, CFD simulation.

radial velocity image is asymmetric. We clearly see the quadrupole and the characteristic outflow, see upper slice. A closer look at the upper slice of Fig. 12 (a) is given in Fig. 12 (b). The radial velocity pattern is similar to the LIDAR measurements. Here we include additional information, namely a vorticity magnitude iso-surface and velocity vectors. We conclude that three vortices are involved in that pattern - the main vortex, the engine vortex, that stays stable due to the landing gears, as well as a the tailplane vortex rebounding from the ground.

\section{CONCLUSION}

This work was dedicated to studying the wake vortex evolution during an aircraft landing with LIDAR and CFD methods. A special emphasis was put on the effects of the landing gear and the effect on the coherent structures in the wake. A very detailed CAD model of the A320 "ATRA" research aircraft, operated by the DLR, was used in those RANS simulations. A high-lift configuration with deflected flaps and slats with and without main and nose landing gears was used. The RANS simulation used a hybrid unstructured mesh with adaptively refined regions behind the wing, flaps, slats, landing gears, and tailplane. The compressible, finite volume DLR TAU Code was used to solve the RANS equations, with the robust and accurate Spalart-Allmaras model. The LES domain was handled by MGLET, an incompressible code employing a forth-order accurate compact finite-volume scheme and a dynamic Lagrangian model for the unresolved scales. The RANS fields were used to initialize the flow-field in the LES domain at suitable height intervals.

Flight measurement data from validation experiments were used to assess the circulation of the wake vortices with this simulation method. Indeed, a good agreement between the circulations resulting from the computation and flight measurements was achieved. The simulations have shown a surprising effect that may be induced by the landing gears. Though their impact on the circulation decay was moderate, a relatively strong coherent structure forms out of the engine - landing gear system whereas the engine wake dissipates quickly in the simulations without landing gears. The Burnham-Hallock model that was used to approximate tangential velocity profiles out of ground, has to be completed by mirror vortices close to the ground. However, this model did not capture the above mentioned engine vortex seen in the simulation with landing gear.

LIDAR measurements from a measurement campaign at Vienna international airport reveal interesting vortex patterns behind various types of aircraft after touchdown. The measured radial velocity patterns obtained by RHI scans could not be explained by just one vortex pair. The measurements were qualitatively compared to simulation results where a similar setup was employed. Indeed, the pattern matched very well. It could be shown that it was generated by a three vortex pair system, the main vortices, the tailplane vortices and the 
engine-gear-system vortices, presented above. This study motivates the usage of LES data for the optimization of LIDAR post-processing algorithms of aircraft wake vortices in ground proximity.

\subsection{Acknowledgments}

The CPU time on the HPC Cluster SuperMUC, provided by the Leibniz Supercomputing Centre (LRZ) as well as the cooperation with AustroControl are greatly acknowledged.

\section{REFERENCES}

[1] Gerz, T., Holzäpfel, F., and Darracq, D., "Commercial aircraft wake vortices," Progress in Aerospace Sciences 38(3), 181-208 (2002).

[2] Stephan, A., Holzäpfel, F., and Misaka, T., "Hybrid simulation of wake-vortex evolution during landing on flat terrain and with plate line," International Journal of Heat and Fluid Flow 49, 18-27 (2014).

[3] Constant, G. D. J., Foord, R., Forrester, P. A., and Vaughan, J. M., "Coherent laser radar and the problem of aircraft wake vortices," Journal of Modern Optics 41, 2153-2173 (1994).

[4] Smalikho, I. N., Banakh, V., Holzäpfel, F., and Rahm, S., "Method of radial velocities for the estimation of aircraft wake vortex parameters from data measured by coherent doppler lidar," Optics Express 23, A1194-A1207 (2015).

[5] Banakh, V. and Smalikho, I. N., [Coherent Doppler WInd Lidars in a Turbulent Atmosphere], Artech House, Boston \& London (2013).

[6] Centaur, "Centaursoft: Centaur grid generation software," (2017).

[7] Kroll, N., Becker, K., Rieger, H., and Thiele, F., [MEGADESIGN and MegaOpt - German Initiatives for Aerodynamic Simulation and Optimization in Aircraft Design] (2009).

[8] Spalart, P. and Allmaras, S., "A one-equation turbulence model for aerodynamic flows," in [30th Aerospace Sciences Meeting and Exhibit, Aerospace Sciences Meetings], (1992).

[9] Bier, N., Rohlmann, D., and Rudnik, R., "Numerical maximum lift predictions of a realistic commercial aircraft in landing configuration," in [AIAA 2012-0279, 50th AIAA Aerospace Sciences, Nashville, USA], (2012).

[10] Rohlmann, D. and Keye, S., "Stall maneuver simulation of an elastic transport aircraft based on flight test data," in [AIAA 2015-2570, AIAA Aviation, 33rd AIAA Applied Aerodynamics Conference, Dallas, USA], (2015).

[11] Rumsey, C. L., Long, M., Stuever, R. A., and Wayman, T. R., "Summary of the first aiaa cfd high-lift prediction workshop," Journal of Aircraft 48(6), 2068-2079 (2011).

[12] Manhart, M., "A zonal grid algorithm for dns of turbulent boundary layers," Computer and Fluids 33(3), $435-461$ (2004).

[13] Meneveau, C., Lund, T. S., and Cabot, W. H., "A lagrangian dynamic subgrid-scale model of turbulence," Journal of Fluid Mechanics 319, 353-385 (1996).

[14] Hokpunna, A. and Manhart, M., "Compact fourth-order finite volume method for numerical solutions of navier-stokes equations on staggered grids," Journal of Computational Physics 229(20), 7545-7570 (2010).

[15] Misaka, T., Holzäpfel, F., and Gerz, T., "Large-eddy simulation of aircraft wake evolution from roll-up until vortex decay.," AIAA Journal 53, 2646-2670 (2015).

[16] Fujii, K., "Unified zonal method based on the fortified solution algorithm," Journal of Computational Physics 118, 92-108 (1995).

[17] Kalnay, E., [Atmospheric Modeling, Data Assimilation and Predictability], Cambridge University Press (2003).

[18] Stephan, A., Holzäpfel, F., and Zholtovski, S., "The effect of gusts on aircraft wake vortices," Aircraft Engineering and Aerospace Technology 89, 692-702 (2017).

[19] Smalikho, I. and Rahm, S., "Lidar investigations of the effects of wind and atmospheric turbulence on an aircraft wake vortex," Journal of Atmospheric and Oceanic Technology 23 No.2, 137-146 (2010). 